\title{
Longitudinal Space-Charge Geometric Factor for an Elliptical Beam
}

\author{
S. Koscielniak, TRIUMF*, 4004 Wesbrook Mall, Vancouver, B.C., V6T 2A3, Canada
}

\begin{abstract}
The longitudinal space-charge impedance for a particle beam, for which the azimuthal extent is much longer than the transverse, is typically expressed[3] in terms of a geometrical factor, $g_{0}$, which depends on the transverse charge distribution and the geometry of the vacuum chamber. Expressions for $g_{0}$ for a round beam in a concentric round pipe are well known[3,7]; and values may be given for the onaxis impedance or for the impedance ensemble-averaged over the beam cross-section. We have obtained analogous expressions for the on-axis and ensemble-average factors $g_{0}$ for the case of an elliptical charge distribution $\rho(m, x, y)=\left[1-(x / a)^{2}-(y / b)^{2}\right]^{m}$ inside a confocal elliptic metallic pipe for the indices $m=0,1,2$. However, our results are not completely general; for example, the pipe cannot have the same eccentricity as the beam.
\end{abstract}

\section{INTRODUCTION}

\subsection{Uniform elliptic beam in metallic enclosure}

Given the 2D transverse electrical potential of a charge distribution with a metallic boundary condition, it is a straight-forward matter to write down a longitudinal geometric factor. Laslett $[5,6]$ gave the direct and image potentials for uniform elliptical beams enclosed by a variety of boundaries from flat plates to hyperbolic pole-faces; and these results could easily have been used to write $g_{0}$ factors circa 1977. [One result[4] does not satisfy $\nabla \wedge \mathbf{E}=\mathbf{0}$.]

\subsection{Non-uniform elliptic beam in free space}

As noted by Kellogg[1], and Houssais and Sacherer [2], for the case of free space in two-dimensions, the field in the interior of a disc with elliptical equi-density contours $\rho(m, x, y)$ where $m>0$ is an integer, may be evaluated from definite integrals. For example the uniform ellipse with unit charge, $m=0$, has fields:

$$
E_{x}=\frac{x}{a(a+b) \pi} \quad \text { and } \quad E_{y}=\frac{y}{b(a+b) \pi} .
$$

The fields in the case $m=1$ have previously been given by Lapostolle [8,9] et al. Similar problems but with a confocal elliptic conducting boundary are much more difficult and have to be treated by solving the Poisson equation.

\subsection{Poisson's equation}

It is natural to solve an electrostatics problem by finding a potential $\Phi$ which satisfies Poisson's equation $\nabla^{2} \Phi=-\rho$

\footnotetext{
* Work supported via a contribution agreement through the National Research Council of Canada.
}

and then forming $\mathbf{E}=-\nabla \Phi$. Solution of the Poisson equation is facilitated by adopting a coordinate system whose level surfaces coincide with the boundary of the charge distribution and or a metallic enclosing boundary.

\subsection{Elliptic confocal coordinates}

We consider an ellipse with major and minor semi-axes $a$ and $b$, respectively; and with foci at $\pm c$ where $c^{2}=a^{2}-b^{2}$. We adopt elliptic coordinates $u, v$ which are related to the cartesian coordinates $x, y$ as follows:

$$
x=c \cosh (u) \cos (v) \text { and } y=c \sinh (u) \sin (v) .
$$

The level surfaces of constant $u$ and constant $v$ are families of ellipses and hyperbolae, respectively, with common foci. These level surfaces are sketched in figure 1. The ellipse

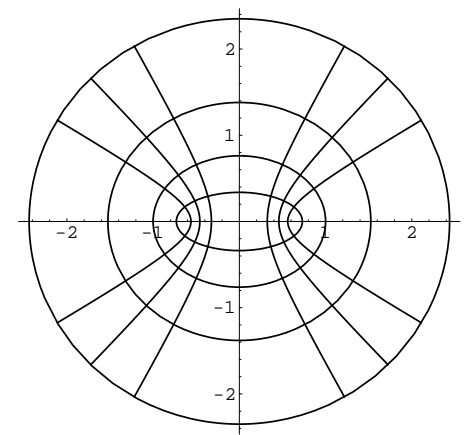

Figure 1: Level surfaces $u=1 / 2,1,3 / 2,2$ and $v=$ $\pm \pi / 6, \pm \pi / 4, \pm \pi / 3$, etc. for $u_{b}=1$.

with major and minor semi-axes $a, b$ is given by $\tanh u=$ $b / a$ and we denote this value

$$
u_{b}=\frac{1}{2} \log _{e}\left[\frac{a+b}{a-b}\right]=\ln \left[\frac{a+b}{c}\right] .
$$

Similarly, the wall ellipse with semi-axes $p, q$ is given by $u_{w}=\ln [(p+q) / c]$ or $\tanh u_{w}=q / p$ (where $\left.p>q\right)$.

Let $h^{2}=c^{2}\left(\sinh ^{2} u+\sin ^{2} v\right)$. In the elliptic coordinates, Poisson's equation becomes:

$$
\left[\frac{\partial^{2}}{\partial u^{2}}+\frac{\partial^{2}}{\partial v^{2}}\right]=-h^{2}(u, v) \rho(u, v) .
$$

First consider the homogeneous equation when $\rho \equiv 0$. Let us introduce the dual-complex symbols $i^{2}=-1$ and $j^{2}=+1$ and identify the four directions $1, i, j, i j$. Possible solutions of $\nabla^{2} \Phi=0$ are then the components of

$$
e^{j u} e^{i v}=(\cosh u+j \sinh u)(\cos v+i \sin v) .
$$

Other possible solutions are $\Phi=1, u, v$; and $\Phi=u$ is of particular interest because it is the far-field solution analogous to $\ln r$ in polar coordinates. 


\section{UNIFORM ELLIPTIC BEAM}

We treat the case of constant density normalized to unit charge, $\rho=1 / \pi a b$. We follow, basically, the exposition of Symon [10] excepting that we shall give a correct expression for the field in the exterior region, as is essential to computing the geometric factor. The particular integral is

$$
\text { P.I. }=-[\cosh (2 u)+\cos (2 v)] /\left[4 \pi \sinh \left(2 u_{b}\right)\right] .
$$

We must now find the interior complementary function and the potential exterior to the beam $\Phi_{\text {ext }}$; both depend on whether it is free space or a metallic boundary.

\subsection{Free space}

The potential functions are

$$
\begin{aligned}
\Phi_{\text {int }} & =P . I .+A_{1} \cosh (2 u) \cos (2 v) \\
\Phi_{\text {ext }} & =B_{1}[\cosh (2 u)-\sinh (2 u)] \cos (2 v)+B_{2} \times u
\end{aligned}
$$

The azimuthal (i.e. $v$-dependence) of $\Phi_{\text {ext }}$ vanishes as $r \rightarrow \infty$ leaving only the logarithmic term $u \rightarrow \ln (r)$, as desired. The adjustable coefficients $A_{1}, B_{1}, B_{2}$ are found from the continuity of the field components $E_{u}, E_{v}$ across the boundary $u=u_{b}$. The field is

$$
\mathbf{E}=-\nabla \Phi=-\frac{1}{h}\left[\mathbf{e}_{u} \frac{\partial}{\partial u}+\mathbf{e}_{v} \frac{\partial}{\partial v}\right] \Phi .
$$

Because the field component $E_{v}\left(u_{b}, v\right)$ tangential to the surface $u=u_{b}$ is not zero, so it follows that the boundary of the charge distribution is not an equipotential.

The result for the field in the interior of the disc is found to be identical with the components given above (1). The field exterior to the disc is given by

$$
E_{x}=x \frac{[1-\tanh u]}{(a-b)(a+b) \pi}, \quad E_{y}=y \frac{[\operatorname{coth} u-1]}{(a-b)(a+b) \pi} .
$$

\subsection{Elliptic metallic boundary}

We introduce a grounded, confocal elliptical conducting wall along the level surface $u=u_{w}>u_{b}$. The particular integral is unchanged, but we shall have to find a new complementary function and exterior potential to satisfy the condition $\mathbf{E}=\mathbf{0}$ at the wall. We take:

$$
\begin{aligned}
\Phi_{\text {int }} & =\text { P.I. }+A_{1} \cosh (2 u) \cos (2 v), \\
\Phi_{\text {ext }} & =B_{1} \cos (2 v) \cosh \left(2 u-2 u_{w}\right)+B_{2} \times\left(u-u_{w}\right),
\end{aligned}
$$

where we have arranged $\Phi_{\text {ext }}$ to be zero at the wall. Continuity of the field across the boundary of the charge distribution $\left(u=u_{b}\right)$ determines the adjustable constants. For the interior region, cartesian components of the field are:

$$
E_{x}=x \frac{\left[-b+a \tanh \left(2 u_{w}\right)\right]}{a(a+b)(a-b) \pi}, E_{y}=y \frac{\left[+a-b \tanh \left(2 u_{w}\right)\right]}{b(a+b)(a-b) \pi},
$$

which clearly shows that the induced charges affect the field in the region enclosed by the conductor. Evaluation of the formulae is facilitated by noting that $\tanh \left(2 u_{w}\right)=$ $2 p q /\left(p^{2}+q^{2}\right) \approx 1$. The confocal condition implies $c^{2}=p^{2}-q^{2}$ for the wall ellipse.

\subsection{Geometrical factor}

To make $\Phi$ continuous at $u=u_{b}$ we add a constant to $\Phi_{\text {int }}$. Because $\Phi_{\text {ext }}\left(u_{w}\right)=0$ it follows that the line integral $\int \mathbf{E} \cdot d \mathbf{l}$ from any point $u, v$ within the charged disc to any point on the wall is simply $\Phi_{\text {int }}(u, v)$. From this it follows that the space-charge geometrical factor is directly proportional to $\Phi_{\text {int }}(u, v)$.

On-axis $g_{0} \quad$ Because of peculiar behaviour of the elliptic coordinate system as $u \rightarrow 0$, the beam centre is located at $(u=0, v= \pm \pi / 2)$. Thus the on-axis impedance is

$$
\begin{gathered}
g_{0}=4 \pi \Phi_{\text {int }}(0, \pi / 2)=2\left(u_{w}-u_{b}\right)+\tanh \left(2 u_{w}\right) . \\
\text { Simplifying: } g_{0}=2 \ln \left[\frac{p+q}{a+b}\right]+\frac{2 p q}{\left(p^{2}+q^{2}\right)} .
\end{gathered}
$$

The logarithmic term contains essentially the quotient of the average radii of each of the two ellipses; and the algebraic term in $p, q$ is approximately 1 . Hence this result is very similar to the "classical" formula $g_{0}=1+2 \ln (p / a)$ for a uniform circular beam and pipe.

Ensemble average $g_{0}$ The on-axis space-charge impedance gives the "worst case scenario". A more representative value is given by forming the ensemble average over the beam cross-section.

$$
\left\langle g_{0}\right\rangle=4 \pi \int_{0}^{u_{b}} \int_{-\pi}^{+\pi} \Phi_{\text {int }} \rho h^{2} d u d v .
$$

For the case of constant density, the ensemble average is

$\left\langle g_{0}\right\rangle=2\left(u_{w}-u_{b}\right)+\frac{1}{2} \tanh \left(2 u_{w}\right)=2 \ln \left[\frac{p+q}{a+b}\right]+\frac{p q}{\left(p^{2}+q^{2}\right)}$.

This expression is very similar to the ensemble geometric factor $\left\langle g_{0}\right\rangle=2 \ln (p / a)+(1 / 2)$ for a round beam and pipe.

\section{BEAM WITH PARABOLIC DENSITY}

We now consider the slightly more complicated case that the charge distribution is $\rho(1, x, y)$. The particular integral:

$$
\begin{aligned}
\text { P.I. } & =A_{1}[\cosh 2 u+\cos 2 v]+A_{2}[\cosh 4 u+\cos 4 v] \\
& +A_{3}[\cosh 4 u \cos 2 v+\cos 4 v \cosh 2 u]
\end{aligned}
$$

We substitute into the Poisson equation and compare coefficients of $\cos (4 v) \cosh (2 u)$, $\cosh 4 u$, and $\cosh 2 u$ to obtain three equations for the adjustable constants.

\subsection{Free space}

We take the potential functions:

$$
\begin{aligned}
\Phi_{\text {int }} & =\text { P.I. }+A_{1} \cosh (2 u) \cos (2 v)+A_{2} \cosh (4 u) \cos (4 v) \\
\Phi_{\text {ext }} & =B_{1} \cos (2 v)[\cosh (2 u)-\sinh (2 u)] \\
& +B_{2} \cos (4 v)[\cosh (4 u)-\sinh (4 u)]+B_{3} u .
\end{aligned}
$$

Continuity of the field components $E_{u}, E_{v}$ at the boundary of the charge distribution $\left(u=u_{b}\right)$ determines the adjustable constants $A_{1}$ through $B_{3}$. 


\subsection{Elliptic metallic boundary}

We introduce a grounded, confocal elliptical conducting wall along the level surface $u=u_{w}>u_{b}$. We take:

$$
\begin{aligned}
\Phi_{\text {int }} & =\text { P.I. }+A_{1} \cosh (2 u) \cos (2 v)+A_{2} \cosh (4 u) \cos (4 v) \\
\Phi_{\text {ext }} & =B_{1} \cos (2 v) \cosh \left[2\left(u-u_{w}\right)\right] \\
& +B_{2} \cos (4 v) \cosh \left[4\left(u-u_{w}\right)\right]+B_{3} \times\left(u-u_{w}\right) .
\end{aligned}
$$

where we have arranged $\Phi_{\text {ext }}$ to be zero at the wall. Continuity of the field across the boundary determines the adjustable constants. To make $\Phi$ continuous at $u=u_{b}$ we add a constant to $\Phi_{\text {int }}$.

On-axis geometric factor The geometric factor is

$$
g_{0}=2\left(u_{w}-u_{b}\right)+\frac{\left(9 \sinh 6 u_{w}-7 \sinh 2 u_{w}\right)}{6\left(\cosh 2 u_{w}+\cosh 6 u_{w}\right)} \text {. }
$$

This and subsequent expressions for $g_{0}$ contain the same logarithmic term $\left(u_{w}-u_{b}\right)=\ln [(p+q) /(a+b)]$ as in (15); this term represents the "far field" which is independent of the charge distribution so long as it is elliptic. However, the "near field" (in the exterior region) does depend on the details of the charge distribution and feeds into the hyperbolic functions term.

Ensemble average geometric factor After performing the integral (16) the result is

$$
\left\langle g_{0}\right\rangle=2\left(u_{w}-u_{b}\right)+\frac{\left(33 \sinh 6 u_{w}-31 \sinh 2 u_{w}\right)}{\left[72 \cosh 2 u_{w} \cosh 4 u_{w}\right]} \text {. }
$$

\section{BEAM WITH QUARTIC DENSITY}

We now consider the more complicated case that the charge distribution is $\rho(2, x, y)$. Because we are only interested here in the geometric factor, we shall only consider the case of a metallic enclosure and not the free-space case. The particular integral is:

$=A_{1}(\cosh 2 u+\cos 2 v)+A_{4}(\cosh 4 u \cos 2 v+\cos 4 v \cosh 2 u)$

$+A_{2}(\cosh 4 u+\cos 4 v)+A_{5}(\cosh 6 u \cos 2 v+\cos 6 v \cosh 2 u)$

$+A_{3}(\cosh 6 u+\cos 6 v)+A_{6}(\cosh 6 u \cos 4 v+\cos 6 v \cosh 4 u)$

After substitution into the Poisson equation, comparison of the coefficients of $\cos 6 v \cosh 4 u, \cos 6 v \cosh 2 u$, $\cos 4 v \cosh 2 u, \cosh 6 u, \cosh 4 u, \cosh 2 u$ gives simple equations for $A_{6}, A_{5}, A_{4}, A_{3}, A_{2}, A_{1}$, respectively.

\subsection{Elliptic metallic boundary}

We now find the interior complementary function and the exterior potential function.

$$
\begin{aligned}
& \Phi_{\text {int }}=\text { P.I. }+A_{1} \cosh (2 u) \cos (2 v)+A_{2} \cosh (4 u) \cos (4 v) \\
&+A_{3} \cosh (6 u) \cos (6 v) \\
& \Phi_{\text {ext }}=B_{1} \cos (2 v) \sinh \left[2\left(u-u_{w}\right)\right]+B_{4}\left(u-u_{w}\right) \\
&+B_{2} \cos (4 v) \sinh \left[4\left(u-u_{w}\right)\right]+B_{3} \cos (6 v) \sinh \left[6\left(u-u_{w}\right)\right]
\end{aligned}
$$

The adjustable coefficients are determined by the continuity of $\mathbf{E}$ across the charge boundary at $u=u_{b}$. For the purpose of evaluating $g_{0}$, we make the potential continuous across the boundary $u_{b}$ by adding a constant to $\Phi_{\text {int }}$.

\section{On-axis geometric factor}

$$
\begin{aligned}
g_{0} & =2\left(u_{w}-u_{b}\right) \\
& +\frac{\left(127 \sinh \left(2 u_{w}\right)-90 \sinh \left(6 u_{w}\right)+55 \sinh \left(10 u_{w}\right)\right]}{60\left[-1+2 \cosh \left(4 u_{w}\right)\right] \cosh \left(2 u_{w}\right) \cosh \left(4 u_{w}\right)}
\end{aligned}
$$

\section{Ensemble average geometric factor}

$$
\begin{aligned}
\left\langle g_{0}\right\rangle & =2\left(u_{w}-u_{b}\right) \\
& +\frac{\left[986 \sinh \left(2 u_{w}\right)-675 \sinh \left(6 u_{w}\right)+365 \sinh \left(10 u_{w}\right)\right]}{600\left[-1+2 \cosh \left(4 u_{w}\right)\right] \cosh \left(2 u_{w}\right) \cosh \left(4 u_{w}\right)}
\end{aligned}
$$

\section{CONCLUSION}

We have found the electrical potential for an elliptical charge distribution $\rho \propto\left[1-(x / a)^{2}-(y / b)^{2}\right]^{m}$ within a confocal elliptical metallic boundary for the cases $m=$ $0,1,2$ and have obtained the corresponding on-axis and ensemble-average geometric factors $g_{0}$. We have noted the influence of induced charges on the grounded conductor upon the fields within, by comparison of the potentials with the free-space case of no boundary. This paper is a much abridged version of a TRIUMF design note[11] which contains all the mathematical details and more discussion of $2 \mathrm{D}$ electrostatics problems. Formulae were generated with the aid of MATHEMATICA[12].

\section{REFERENCES}

[1] Kellogg: Foundations of potential theory, Dover Publications, New York, 1953.

[2] F.J. Sacherer: R.M.S. envelope equations with space charge, IEEE Trans. Nucl. Sci. Vol.18 No.3, pg.1105, (1971).

[3] A. Hofmann: Single-beam collective phenomena-longitudinal, CERN 77-13, pp. 141-144.

[4] L.J. Laslett: PEP-91 (1974), or CERN 77-13, pg.322.

[5] L.J. Laslett: The image-field potential of a uniformly-charged ellipse..., in "Selected Works of L. Jackson Laslett", LBL PUB-616, Vol.III (1987) pp. 3-181 to 3-279.

[6] L.J. Laslett: Potential of a uniformly charged beam with an elliptical shape, LBL PUB-616, Vol.II (1987) pg. 3-113.

[7] R. Baartman: Form factor $g$ in longitudinal space charge impedance, TRI-DN-K207, 1992.

[8] P. Lapostolle, A. Lombardi, T. Wangler: A model of nonlinear space-charge forces in a charged particle beam, CERN/PS 93-11 (HI).

[9] T.P. Wangler et al.: Space-charge induced emittance growth in an elliptical charged particle beam, Proc. 1995 Particle Accelerator Conf., Washington D.C., IEEE Pres, pg. 3606.

[10] E. Crosbie and K. Symon: AIP Conference Proceedings 377, Space-charge dominated beams and applications of high brightness beams, Bloomington, Indiana, 1995, pg. 260.

[11] S. Koscielniak: Longitudinal space-charge geometric factor for an elliptical beam..., TRI-DN-00-30, 2000.

[12] S. Wolfram: Mathematica - a system for doing mathematics by computer, Addison Wesley Publishing Co. 